\title{
A fully-active fine-grained detector with three readout views
}

A. Blondel, ${ }^{a}$ F. Cadoux, ${ }^{a}$ S. Fedotov, ${ }^{b}$ M. Khabibullin, ${ }^{b}$ A. Khotjantsev, ${ }^{b}$ A. Korzenev, ${ }^{a}$ A. Kostin, ${ }^{b}$ Y. Kudenko, ${ }^{b, d, e}$ A. Longhin, ${ }^{c}$ A. Mefodiev, ${ }^{b}$ P. Mermod, ${ }^{a}$ O. Mineev, ${ }^{b}$ E. Noah, ${ }^{a}$ D. Sgalaberna, ${ }^{a, *}$ A. Smirnov, ${ }^{b}$ N. Yershov ${ }^{b}$

\author{
${ }^{a}$ University of Geneva, Geneva, Switzerland \\ ${ }^{b}$ Institute for Nuclear Research of the Russian Academy of Sciences, Moscow, Russia \\ ${ }^{c}$ INFN Padova, Italy \\ ${ }^{d}$ Moscow Institute of Physics and Technology, Moscow Region, Russia \\ ${ }^{e}$ Moscow Engineering Physics Institute (MEPhI), Moscow, Russia \\ *Now at CERN \\ E-mail: davide.sgalaberna@cern.ch
}

\begin{abstract}
Aвstract: This paper describes a novel idea of a fine-grained fully-active plastic scintillator detector made of many optically independent $1 \times 1 \times 1 \mathrm{~cm}^{3}$ cubes with readout on three orthogonal projections by wavelength shifting fibers. The original purpose of this detector is to serve as an active neutrino target for the detection, measurement and identification of the final state particles down to a few tenths MeV kinetic energies. The three readout views as well as the fine granularity ensure powerful localization and measurement of the deposited energy combined with good timing properties and isotropic acceptance. The possible application as a new active target for the T2K near detector, initial simulation studies and R\&D test results are reported.
\end{abstract}

Keywords: Neutrino detectors, Scintillators, Particle tracking detectors, Photon detectors for UV, visible and IR photons (vacuum) (photomultipliers, HPDs, others)

ArXIV ePrint: 1707.01785

${ }^{1}$ Corresponding author. 


\section{Contents}

1 Introduction 1

2 The design of the detector 2

3 Measurements 5

4 Simulations $\quad 7$

5 Conclusions $\quad 8$

6 Acknowledgements 9

\section{Introduction}

Plastic scintillator material is very commonly used in high-energy physics and astroparticle physics, providing detectors with good timing properties (often below $1 \mathrm{~ns}$ resolution) and measurement of the deposited energy. The advent of scintillating fibers and/or wavelength shifting fiber readout allows for very flexible geometrical and tracking properties. Neutrino experiments have used scintillators quite systematically, recent examples being the MINOS [1], Minerva [2] and the near detectors suite (ND280) of T2K [3, 4]. In the case of ND280, the readout with silicon photomultipliers was applied systematically to $60^{\prime} 000$ channels, allowing a more compact geometry to be achieved.

In the above examples, narrow plastic scintillator bars are disposed perpendicularly to the neutrino beam direction, a geometry that is suitable for neutrino interactions in beams of energy above typically a few $\mathrm{GeV}$, for which the leading final state particles are predominantly emitted in the forward direction. For lower neutrino energies, the final state lepton of the neutrino charged current interactions is emitted more isotropically. In that case it is interesting to aim at a more isotropic geometry. At all energies, nevertheless, nuclear effects commensurate to the binding energy of the Carbon nucleus or its Fermi Momentum, occurring either in the initial state or in the final state of the neutrino interaction, affect the energy balance and the energy reconstruction [5]. It is thus important to be able to study the effect of nuclear activity by locating the energy deposited by additional nucleons originating from the interaction or resulting from nuclear breakup. In this context the natural granularity scale is around $1 \mathrm{~cm}$, corresponding to the range in plastic of protons with momentum commensurate with the Fermi motion of about $220 \mathrm{MeV} / \mathrm{c}$. Another example of application of a more isotropic geometry could be the astroparticle physics experiments where the detector orbits around the Earth and can detect particles produced by several different sources and coming from any direction.

In case of a detector with scintillator bars disposed perpendicularly to the beam axis (hereinafter this axis referred to as $Z$ ), acceptance and resolution are highly direction-dependent: a particle 
traveling along a single scintillator bar cannot be tracked and the momentum cannot be defined. Furthermore, in a realistic situation several tracks can be produced and it often happens that the energy deposited cannot be uniquely identified and assigned to a particular spatial direction. In this case a three-dimensional readout of the signals will ensure a more isotropic acceptance and reconstruction.

In this paper, concentrating on the case of the project of the ND280 detector upgrade, we present an attempt at such a 3-D design, keeping in mind the need to have the number of channels to a reasonable value and potential applications to other fields in physics.

This article is organized as follows: the detector concept and design is described in Sec. 2; in Sec. 3 the measurements performed on a small prototype with cosmic particles is shown; finally in Sec. 4 the simulation results of the proposed detector are described.

\section{The design of the detector}

The goal of the currently running long baseline experiments, $\mathrm{T} 2 \mathrm{~K}$ and $\mathrm{NO} v \mathrm{~A}$ [6], is to measure the $\mathrm{CP}$ violating phase in the neutrino sector, by measuring neutrino appearance phenomena, such as the $v_{\mu} \rightarrow v_{e}$ and $\bar{v}_{\mu} \rightarrow \bar{v}_{e}$ transitions. To this effect, one compares the neutrino event rate at a near detector, before oscillations occur, with the neutrino event rate at the far detector, whose position, in the case of $\mathrm{T} 2 \mathrm{~K}$ and $\mathrm{NO} v \mathrm{~A}$, is located near the oscillation maximum. On a longer time scale, new experiments, Hyper-K [7] and DUNE [8], will start searching for CP violation with much larger data sample. For this reason it is timely to develop near detector designs in which as much as possible information is acquired, both by establishing the rate and flavour of neutrino interaction events and by understanding the measurement of neutrino energy - since neutrino energy is the quantity which governs the neutrino oscillations. This latter point requires a detailed knowledge of neutrino interactions. Furthermore, the possible differences between electron- vs muon- (anti)neutrino cross sections are essential for the precise measurement of the appearance oscillation phenomenon. Several experiments $[2,3,6,9]$ are currently measuring the neutrino interaction cross sections with scintillator detectors. However a dedicated effort is required to address the specific needs of the neutrino oscillation program. In the case of T2K, these have been spelled out in the T2K ND280 upgrade program:

- the near detector measurements must cover the full polar angle range for the final state lepton with a well understood acceptance;

- the near detector must be capable of measuring (at least the ratio of) electron and muon neutrino cross sections;

- the near detector should be able to address the issue of nuclear effects and their impact on energy reconstruction.

The detector must also be fully active and the amount of dead material must be minimized, in order to detect all the energy released by the produced particles and reconstruct with precision the energy of the interacting neutrino. Furthermore, in an experiment like T2K, Hyper-K and NOvA it becomes very important to have very similar nuclear targets at the near and far detectors, for instance $\mathrm{H}_{2} \mathrm{O}$ at T2K (Hyper-K) and liquid scintillator at $\mathrm{NO} v \mathrm{~A}$. Organic scintillators, ${ }^{12} \mathrm{C}$-based, fulfill as 
much as possible this requirement. A good compromise would be given by a fine granularity detector, $\sim 1 \mathrm{~cm}$, the range of a proton with the Fermi momentum, with a good acceptance over the full solid angle.

An interesting solution is a full 3D detector [10], that would solve the tracking ambiguity issues. However given the combination of large mass and very fine granularity, a prohibitively large number of readout channels $(O(1 \mathrm{M})$, since it scales with the detector volume) would be required if one would read out individually $\mathrm{cm}$-size cubes, leading to high costs and a large amount of dead material. We propose here an alternative solution with more acceptable costs and less dead material inside the neutrino target.

The proposed detector consists of many cubes of extruded scintillator, each one covered by a reflector and read out along three orthogonal directions by wavelength shifting fibers. The chosen scintillator is a composition of a polystyrene doped with $1.5 \%$ of paraterphenyl (PTP) and $0.01 \%$ of POPOP. The cubes produced by Uniplast, a company in Vladimir, Russia, are covered by a $\sim 50 \mu \mathrm{m}$ thick diffusing layer. Depending on the physics case, different technologies can be used for the reflector to constrain the scintillation light inside the cube. In order to provide good light yield and uniformity in the neutrino target nuclei, the reflector is obtained by etching the scintillator surface in a chemical agent that results in the formation of a white micropore deposit, acting as a diffuser, over a polystyrene surface [11]. Each cube has three orthogonal cylindrical holes of $1.5 \mathrm{~mm}$ diameter drilled along $\mathrm{X}, \mathrm{Y}$ and $\mathrm{Z}$ axes. Three $1.0 \mathrm{~mm}$ wavelength shifting (WLS) fibers, multi-clad Kuraray Y11, are inserted through the holes. The ideal size of each cube is $1 \times 1 \times 1 \mathrm{~cm}^{3}$, providing the required fine granularity. The axes $X, Y$ and $Z$ define respectively the width, the height and the length of the detector and the neutrino beam direction is supposed to be centered along the $Z$ axis. We consider a detector of the size of $1.8 \times 0.6 \times 2.0 \mathrm{~m}^{3}$, i.e. about 2 tons, that would correspond to approximately $59 \mathrm{k}$ readout channels. Should this number prove to be too high, the size of the cubes could be increased for instance up to $2 \times 2 \times 2 \mathrm{~cm}^{3}$, corresponding to only $15 \mathrm{k}$ readout channels. For a given detector size, the number of cubes is inversely proportional to the third power of the cube size, while the number of channels scales as the inverse of the square. A picture of a small prototype is shown in Figure 1. The parameters of the detector and scintillator cubes are shown in Table 1 and 2.

The power of such a detector is given by the three views that would provide a $4 \pi$ acceptance and solve the tracking ambiguity efficiently. Thanks to this novel configuration we expect it will be possible to associate the particle hits to the right track for most of the neutrino events. The third view will help solving the ambiguity providing a great improvement compared to the two-views detectors, where different tracks can share the same hits in the 2D projection. This will be possible also thanks to the fact that $\mathrm{O}(1 \mathrm{GeV})$ neutrino interaction events are usually low multiplicity. Furthermore the track reconstruction itself, where hits are linked together, will help. Some ambiguities are still expected for hits close to the neutrino interaction vertex, within a few cms, where some lowmomentum nucleons could be ejected by the nucleus within a very close range. In this case, thanks to the fine granularity, this detector can provide a precise calorimetry of the energy released, improving the final reconstruction of the neutrino energy.

At the T2K most probable neutrino energy, typically $600 \mathrm{MeV}$, the event multiplicity is low and a full cell-by-cell readout, which would be prohibitive both in terms of number of readout channels and added passive material, is not necessary. Our first investigations show that a system with a 

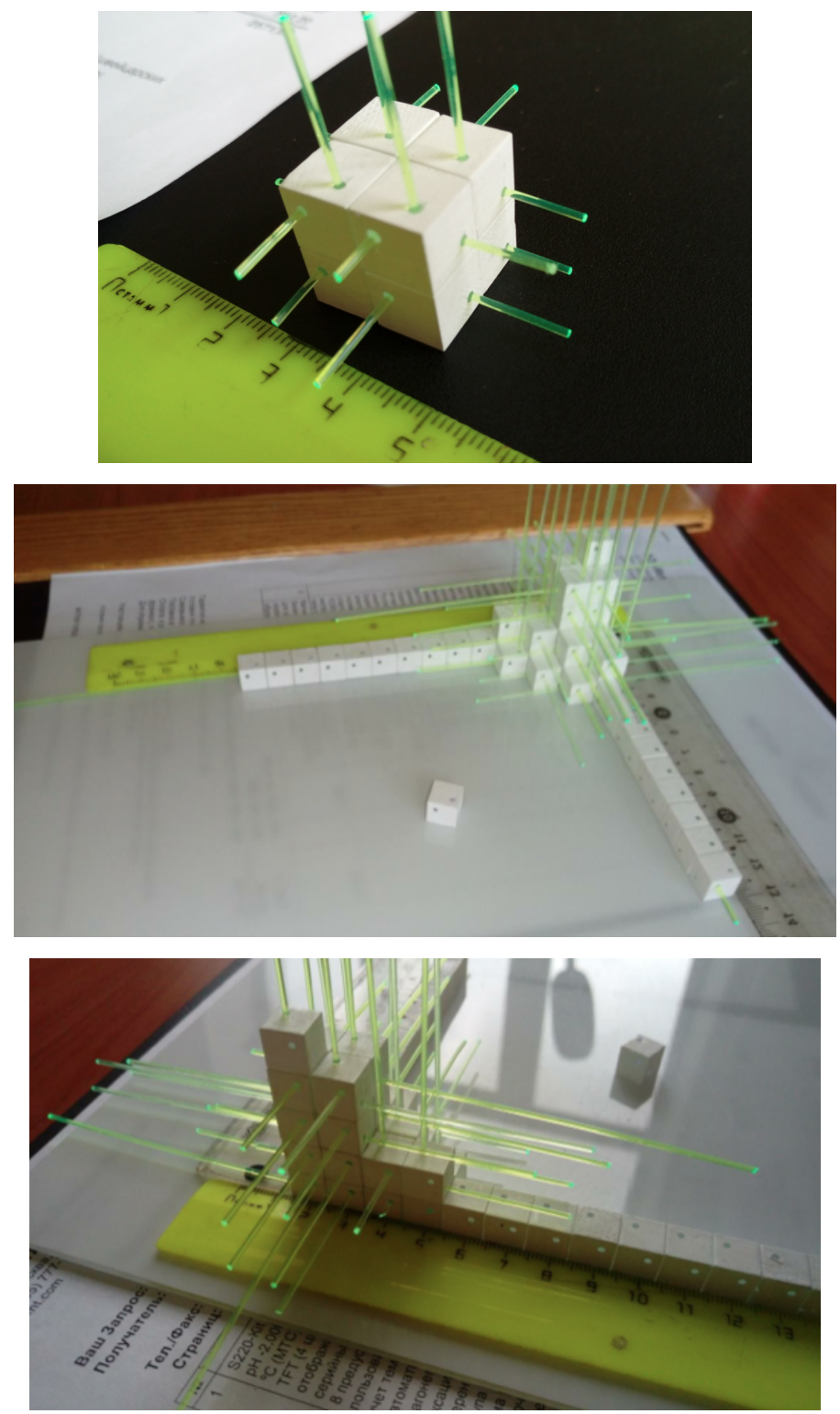

Figure 1. The picture of a small prototype is shown. Several cubes of extruded plastic scintillator with three WLS fibers inserted in the three holes are assembled. The size of each cube is $1 \times 1 \times 1 \mathrm{~cm}^{3}$.

three-view readout looks appropriate. The fine granularity would allow to measure protons with momenta down to $300 \mathrm{MeV} / \mathrm{c}$. None of the above properties could be achieved with a scintillator bar detector. In addition the light is enclosed within the cube and the light yield is expected to be higher than in standard scintillator bars. In this configuration, the energy deposited by a given ionizing particle is simultaneously collected by three WLS fibers, instead of only one, improving pattern recognition and the light yield. Furthermore, in order to obtain three views, the energy deposited by a particle in a single cube is enough. This is a tremendous advantage compared to scintillator bar detectors where two views are provided by a particle depositing energy in at least 
Table 1. Main parameters of the proposed detector of the size of $1.8 \times 0.6 \times 2.0 \mathrm{~m}^{3}$.

\begin{tabular}{c|c|c}
\hline \hline Parameter & Cube edge: $1 \mathrm{~cm}$ & Cube edge: $2 \mathrm{~cm}$ \\
\hline \hline \# of cubes & $2.16 \mathrm{M}$ & $270 \mathrm{k}$ \\
\hline \# of channels & $58.8 \mathrm{k}$ & $14.7 \mathrm{k}$ \\
\hline \hline
\end{tabular}

Table 2. Main parameters of each scintillator cube with $1 \mathrm{~cm}$ edge.

\begin{tabular}{c|c}
\hline \hline Parameter & Value \\
\hline \hline Coating thickness & $50 \mu \mathrm{m}$ \\
\hline Hole diameter & $1.5 \mathrm{~mm}$ \\
\hline WLS fiber diameter & $1.0 \mathrm{~mm}$ \\
\hline \hline
\end{tabular}

two different bars.

This detector is quite useful to distinguish pions and muons from protons and electrons. In addition it is essential for separating the electrons, which are a manifestation of the electron neutrino interactions, from the $\gamma \rightarrow e^{+} e^{-}$background.

This detector could be also used to detect neutrons produced in the neutrino interaction. Indeed an additional coating of either Gadolinium or Lithium could be applied on the surface of each cube, similarly to what has been done for the SOLID experiment [12]: the neutrons are captured after being thermalized and photons, delayed with respect to the lepton produced by the interaction, are released. As already mentioned above, the conceived geometry could be useful also for astroparticle physics experiments, thanks to the full solid angle acceptance.

Different configurations of the detector could be obtained in order to fulfill different requirements, such as an improved angular resolution. In this case half of the plastic cubes could be replaced with a very low density material, e.g. AIREX (with a density of $0.06 \mathrm{~g} / \mathrm{cm}^{3}$, about $6 \%$ density of that of plastic scintillator) allowing tracks to travel for a longer distance and improve their separation. These aspects are not addressed here.

\section{Measurements}

In order to study the performance of the scintillator cubes, we carried out measurements of the light yield produced from cosmic ray muons using a small plastic counter. The test bench for detector measurements is shown in Figure 2. The ionization area within the tested cube was localized to a $8 \times 8 \mathrm{~mm}^{2}$ spot defined by the trigger counter size. The readout of the scintillation light from each cube was provided by a $1.3 \mathrm{~m}$ long double-clad Kuraray Y11 WLS fiber coupled at one end to a photosensor, a Hamamatsu multi-pixel photodiode (MPPC). The sensor S12571-025C [13] consists of an array of 1600 independent $25 \times 25 \mu \mathrm{m}$ avalanche photodiodes (pixels) operating in Geiger mode. The MPPC sensitive area is $1 \times 1 \mathrm{~mm}^{2}$. The photo detection efficiency of this MPPC is about 35\% (3.5 V overvoltage) for green light of $520 \mathrm{~nm}$ as emitted by a Y11 fiber. Signals from MPPCs were amplified by a custom-made preamplifier with a gain of 20 , then sent to the $5 \mathrm{GHz}$ sampling digitizer CAEN DT5742 with 12-bit resolution. The signal charge was calculated as an area of signal waveform normalized to number of photoelectrons. The signal timing was obtained 


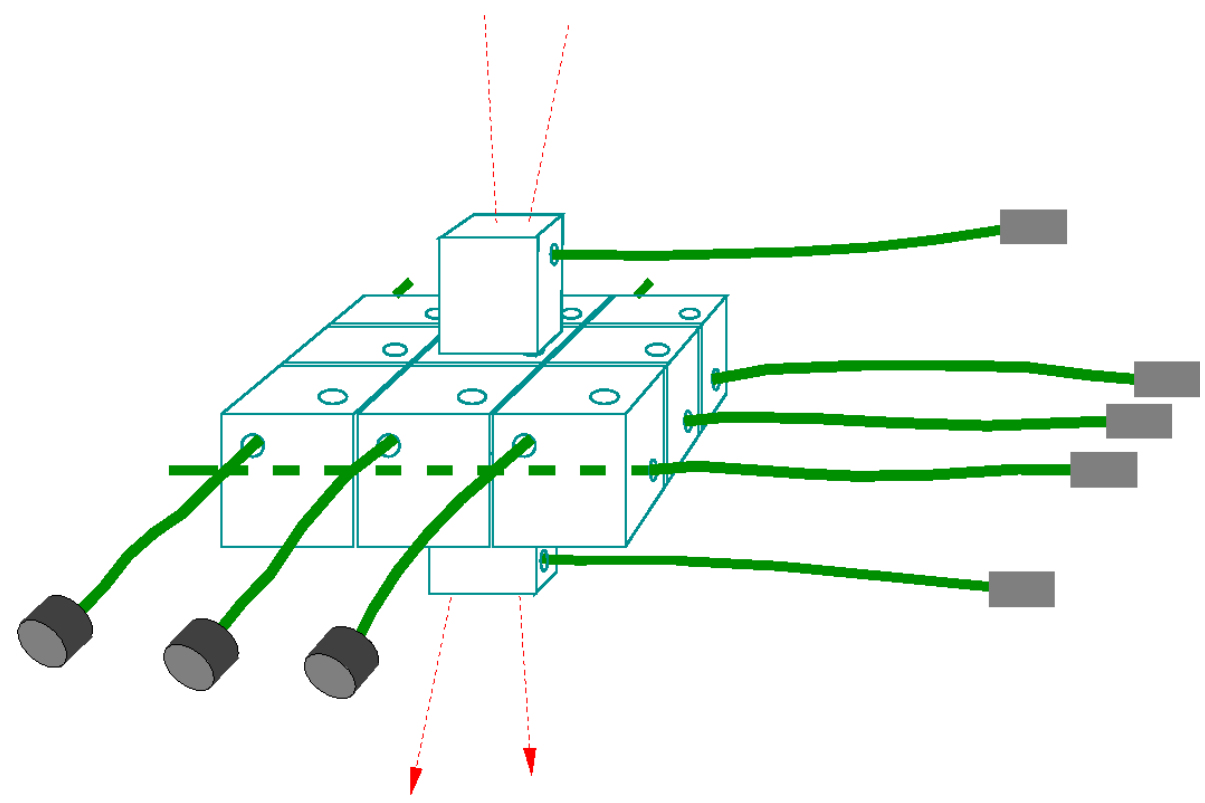

Figure 2. Test bench for study of the parameters of scintillator cubes using cosmic muons. The tested array comprised nine $1 \times 1 \times 1 \mathrm{~cm}^{3}$ cubes. Two trigger counters, each of a $8 \times 8 \mathrm{~mm}^{2}$ cross section, were located above and below the tested cubes.

at the $10 \%$ fraction of the signal amplitude. The position of all cubes along the WLS fibers inserted in the holes was fixed at the distance of $1 \mathrm{~m}$ from the photosensor. In order to increase the light yield, the far end of the fiber was covered by a teflon tape.

The light yield of one scintillator cube in photoelectrons (p.e.) per minimum ionizing particle (MIP) obtained with one fiber is plotted in Figure 3. About 55 p.e./MIP were measured with a $1 \mathrm{~m}$ long WLS fiber. For a fiber length of $2 \mathrm{~m}$ the estimated light yield is expected to be about 35 p.e. according to the attenuation length of Y11 for green light [14].

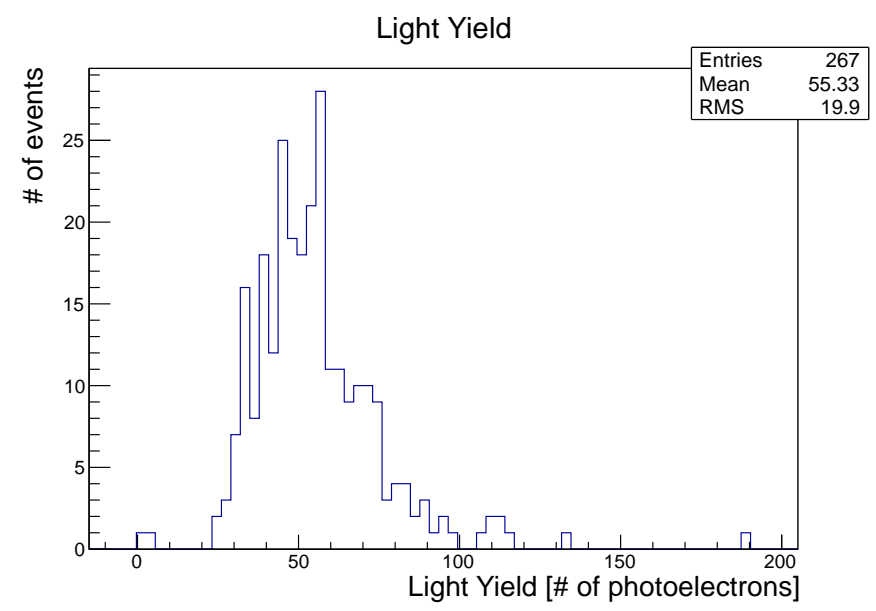

Figure 3. The light yield of a scintillator cube per a minimum ionizing particle with one WLS fiber measured at a distance of $1 \mathrm{~m}$ from the MPPC. 
Since the white chemical reflector does not fully contain the scintillation light, its leak of the scintillating light from one cube to neighboring ones was investigated. The cross-talk was measured between the fired central cube and adjacent cubes which surround the central one as shown in Figure 2. The cross-talk is defined as the ratio of the light yield in an adjacent cube to the signal in the central cube. The MPPC dark noise was measured simultaneously during the test and subtracted from the signal in the adjacent cube. Figure 4 shows that on average less than $3 \%$ of scintillating light penetrates from one cube to another.

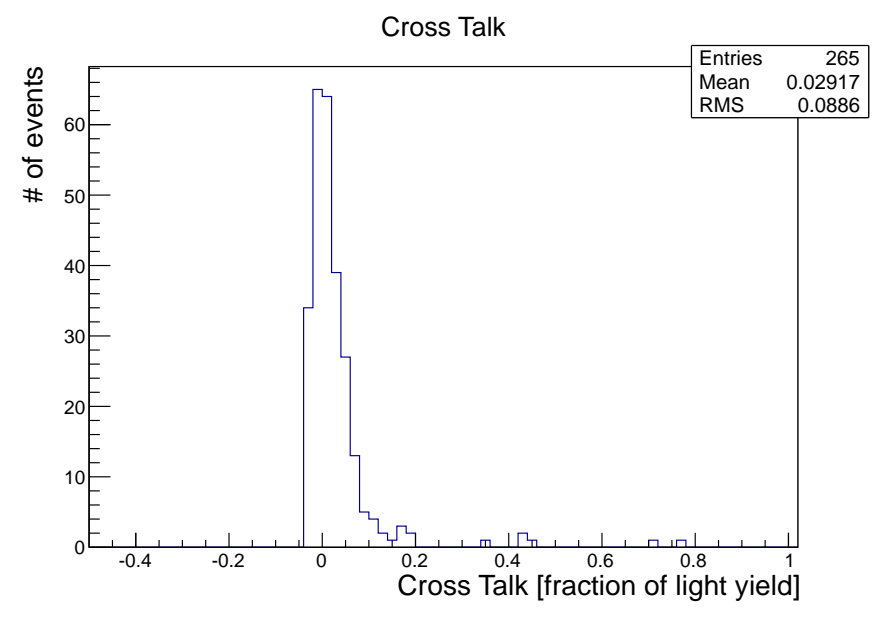

Figure 4. The ratio of the signal in the adjacent cube to the signal in the fired central cube. Negative values are caused by subtracting the average dark noise contribution on the event-by-event basis. Values higher than 0.2 are likely due not to light cross talk but, in large part, to delta-rays or multiple scattering of the cosmic ray in the scintillator cube that produce signal in the adjacent cubes. These entries are taken into account in the final cross talk calculation. The cross-talk is estimated to be $2.9 \%$, that corresponds to the mean of the shown distribution.

In Figure 5 the detector time resolution measured with the setup described above is shown. Thanks to the high light yield provided by this geometry combined with a "fast" readout electronics, we achieve a time resolution of a single MIP particle hit of about $0.91 \mathrm{~ns}$ in a single WLS fiber and about $0.63 \mathrm{~ns}$ for the case when the light is collected by two orthogonal WLS fibers. Further improvement can be obtained by considering the light collected simultaneously by all the three orthogonal WLS fibers. A double-end readout, though more expensive given the doubled number of readout channels, might also improve the time resolution by approximately $40 \%$.

\section{Simulations}

The full detector, corresponding to the parameters in Table 1, has been simulated with the GEANT4 software [15]. The detector response was parametrized and the track reconstruction was performed, for the time being, without pattern recognition. However if there are more tracks in a single event they must fulfill separation criteria in order to be reconstructed. The axes $X, Y$ and $Z$ define respectively the width, the height and the length of the detector. A magnetic field of $0.2 \mathrm{~T}$ has been simulated along $X$, in the same configuration as ND280. About 230k neutrino interactions in the 

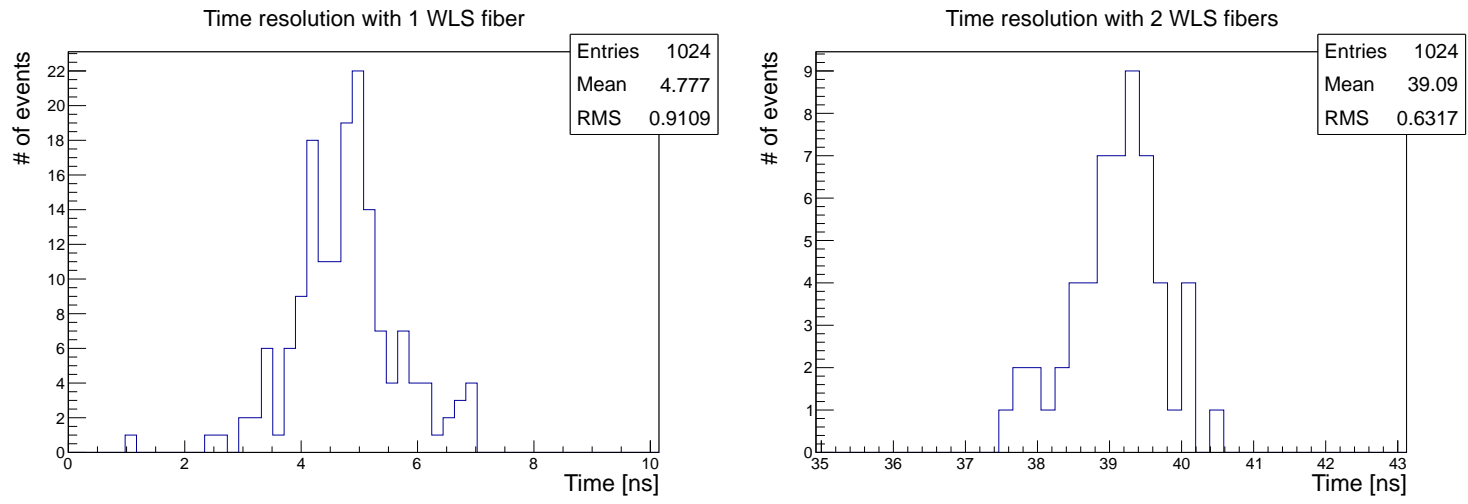

Figure 5. The measured time distribution of the cosmic particle hit in one scintillator cube is shown when the light yield is collected in one WLS fiber (left) and two WLS fibers (right).

detector were simulated with the GENIE software [16] with the neutrino beam centered along the $Z$ axis and the energy spectrum expected at ND280.

The expected performance of the proposed detector was compared to a plastic scintillator detector made of $1 \times 1 \mathrm{~cm}^{2}$ cross-section bars directed along the $X$ and $Z$ direction, whose goal is to measure particles produced at about $90^{\circ}$ with respect to the neutrino beam direction. The results are shown in Figure 6. The reconstruction efficiency for muons is shown as a function of the muon angle with respect to the $Z$ axis. It is clear that the detector proposed in this article has a $4 \pi$ angular acceptance, with a track reconstruction efficiency exceeding $90 \%$ for the whole angular range. Also the reconstruction efficiency as a function of the proton momentum is improved: the momentum threshold to detect protons is reduced from about $450 \mathrm{MeV} / \mathrm{c}$ down to $300 \mathrm{MeV} / \mathrm{c}$. This also shows that in the proposed detector it may be possible to improve the reconstruction of the neutrino energy by measuring also low energy protons and pions.

We confirmed with a dedicated study that the particle identification capability is similar to that of the $X Z$ scintillator bars detector.

\section{Conclusions}

We have shown that the technique of extruded scintillator with wavelength shifting fiber readout can be extended to a three directional readout. The first simulations and the measurements with a small prototype of the conceived detector show encouraging results, with a light yield of more than 50 photo-electrons for one direction. A larger size prototype (aim is $5 \times 5 \times 5 \mathrm{~cm}^{3}$ for a total of 125 cubes and 75 readout channels) is under construction to be exposed to tests at particle beams, in which the timing properties and the possible cross-talk between channels can be evaluated. Further simulation studies will establish the predicted performance, for e.g. electron and photon separation or PID by $\mathrm{dE} / \mathrm{dx}$, to be benchmarked at test beams. A challenging aspect of further design will be the mechanical integration providing sufficient rigidity as well as compact and light disposition of the readout. 

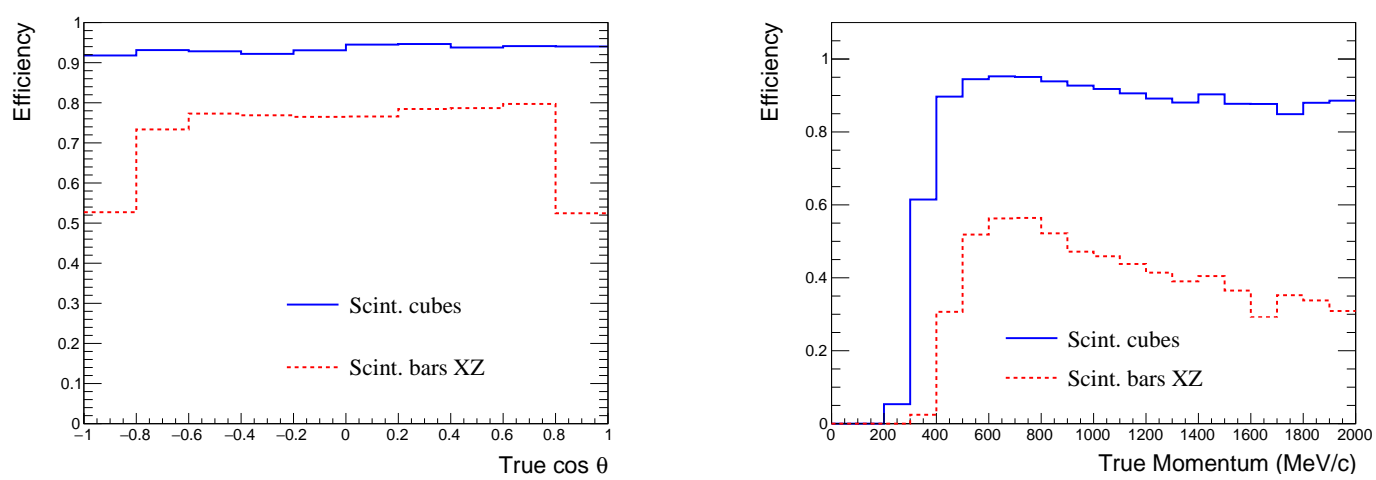

Figure 6. Track reconstruction efficiencies are shown for the particles produced by GENIE neutrino interactions with $10^{21}$ p.o.t. for both the 3-views detector (named SuperFGD) proposed in this article and a plastic scintillator detector made of bars along the $\mathrm{X}$ and $\mathrm{Z}$ directions (named FGD XZ). Left: muon reconstruction efficiency as a function of the truth muon $\cos \theta$. Right: proton reconstruction efficiency as a function of the truth proton momentum.

\section{Acknowledgements}

This work was initiated in the framework of the T2K ND280 upgrade task force, convened by M. Yokoyama and M. Zito. Fruitful discussions in this context with our colleagues from T2K are gratefully acknowledged. D. Sgalaberna was supported by the grant number 200020-172709 of the Swiss National Foundation. The work was supported in part by the RFBR/JSPS grant \# 17-52-50038.

\section{References}

[1] P. Adamson et al., "The MINOS scintillator calorimeter system”, IEEE Trans. Nucl. Sci. 49 (2002) 861-863.

[2] L. Aliaga et al., "Design, Calibration, and Performance of the MINERvA Detector", Nucl.Instrum.Meth. A 743 (2014) 130-159 [arXiv:1305.5199].

[3] K. Abe et al. [T2K Collaboration], “The T2K Experiment”, Nucl. Instrum. Meth. A 659 (2011) 106 [arXiv:1106.1238 [physics.ins-det]].

[4] K. Abe et al., "Proposal for an Extended Run of T2K to $20 \times 10^{21}$ POT", arXiv:1609.04111 [hep-ex].

[5] K. Abe et al., "Measurement of neutrino and antineutrino oscillations by the T2K experiment including a new additional sample of $v_{e}$ interactions at the far detector", arXiv:1707.01048 [hep-ex].

[6] D. Ayres et al., "NOvA Proposal to Build a 30 Kiloton Off-Axis Detector to Study Neutrino Oscillations in the Fermilab NuMI Beamline", arXiv:hep-ex/0503053.

[7] K. Abe et al. [Hyper-Kamiokande Proto- Collaboration], "Physics potential of a long-baseline neutrino oscillation experiment using a J-PARC neutrino beam and Hyper-Kamiokande", PTEP 2015 (2015) 053C02 [arXiv:1502.05199 [hep-ex]]. 
[8] R.Acciarri et al., “ Long-Baseline Neutrino Facility (LBNF) and Deep Underground Neutrino Experiment (DUNE) Conceptual Design Report Volume 2: The Physics Program for DUNE at LBNF", arXiv:1512.06148

[9] T. Koga et al., "Water/CH Neutrino Cross Section Measurement at J-PARC (WAGASCI Experiment)", JPS Conf. Proc. 8 (2015) 023003.

[10] E. Vannuccini et al.,, "CaloCube: A new-concept calorimeter for the detection of high-energy cosmic rays in space”, NIM A 845 (2017) 421-424.

[11] Y. G. Kudenko et al., "Extruded plastic counters with WLS fiber readout", Nucl. Instrum. Meth. A 469 (2001) 340.

[12] Y. Abreu et al., “A novel segmented-scintillator antineutrino detector”, arXiv:1703.01683

[13] http://www.hamamatsu.com/

[14] O. Mineev et al., "Scintillator detectors with long WLS fibers and multi-pixel photodiodes", JINST 6 (2011) P12004 [arXiv:1110.2651 [physics.ins-det]].

[15] J. Allison et al., "Recent developments in Geant4", Nucl. Instrum. Meth. A 835 (2016) 186-225.

[16] C.Andreopoulos et al., "The GENIE Neutrino Monte Carlo Generator", Nucl.Instrum.Meth.A614 (2010) 87-104 